\title{
AUTHORISING OF ANTIPSYCHOTICS IN DEMENTIA BY THE MENTAL WELFARE COMMISSION (MWC) FOR SCOTLAND - A SURVEY OF DESIGNATED MEDICAL PRACTITIONERS (DMPs)
}

\section{Gary Stevenson}

Department of Psychiatry, NHS Fife, Fife, Scotland KY15 5RR (gstevenson@nhs.net)

\section{Background \& Objectives}

- $\quad$ The MWC is a public body responsible for safeguarding the rights and welfare of people in Scotland with mental disorder.

- $\quad$ DMPs are psychiatrists engaged by the MWC to authorise for up to 3 years treatment plans proposed by the patient's psychiatrist (RMO) for persons detained under mental health legislation beyond 8 weeks.

- $\quad$ There are established concerns about antipsychotic safety in dementia with only Risperidone having a (restricted) license in the UK for use in dementia where there are significant behavioural and psychological symptoms of dementia (BPSD)

- To obtain the views and authorising practices of DMPs on antipsychotics for persons with dementia.

\section{Methods}

A questionnaire was emailed twice to all DMPs and also distributed at their national meeting in Winter 2018 to survey their experiences and authorising practises of antipsychotics for BPSD in the preceding 2 years.

\section{Results}

DMP response rate of $31 \%(25 / 81)$

$80 \%$ had authorised antipsychotics for BPSD in the previous 2 years

Only $8 \%$ specified Risperidone, and $8 \%$ had authorised co-prescription of antipsychotics for BPSD

Only $36 \%$ discussed licensing restrictions with the prescribing clinician/RMO and fewer (16\%) included any written information on the prescription authority issued.

$44 \%$ of DMPs imposed time-limits on the authority but with significant variability.

$50 \%$ of DMPs were unsure of their legal accountability for the treatment

authorities that they issued

\section{Conclusions}

- Only a minority of authorising DMPs discuss any of the antipsychotic licensing restrictions with the RMO, and even fewer include any written information on the issued medication authority.

- The MWC should issue guidance to DMPs to minimise variability in DMP authorisation, and clarify the accountability of DMPs for their authorities, particularly for medications authorised out with the product license. 